\title{
Einfluss der thermischen Modifizierung von Holz auf das Wasserrückhaltevermögen (WRV-Wert)
}

\author{
E. Roffael · R. Kraft
}

Eingegangen: 4. November 2010 / Online publiziert: 31. März 2011

(C) The Author(s) 2011. Dieser Artikel ist auf Springerlink.com mit Open Access verfügbar

\section{Influence of thermal wood modification on the Water Retention Value (WRV)}

\begin{abstract}
Thermal modification of wood (ash, beech, spruce) leads to a remarkable decrease in the Water Retention Value (WRV). In case of beech wood the WRV-value decreased after immersion in water for $72 \mathrm{~h}$ from $55.5 \%$ to $40.5 \%$ (about 27\%) and in case of spruce wood from $49.3 \%$ to $34.5 \%$ (about $30 \%$ ). The decrease in the WRV of ash wood was relatively low (about 19\%). The WRV-method seems to be useful to follow up the process of thermal modification of wood.
\end{abstract}

\section{Ziel der Untersuchungen}

Die Modifizierung von Holz durch thermische Behandlung, die das Holz chemisch (Fengel 1966a, 1966b; Windeisen et al. 2007) und physikalisch (Kollmann und Schneider 1963) verändert, ist inzwischen eine industriell praktizierte Technologie. Hierdurch erhöhen sich die Dimensionsstabilität des Holzes und seine Resistenz gegenüber dem biologischen Abbau. Auch das Sorptionsvermögen gegenüber Feuchte wird durch die thermische Behandlung erheblich eingeschränkt (vgl. Ewert und Scheiding 2005; Hill 2006).

E. Roffael ( $₫)$

Georg-August-Universität Göttingen, Büsgenweg 1,

37077 Göttingen, Deutschland

e-mail: eroffae1@gwdg.de

R. Kraft

Büsgen-Institut, Abteilung Molekulare Holzbiotechnologie und Technische Mykologie, Georg-August-Universität Göttingen, Büsgenweg 2, 37077 Göttingen, Deutschland
Zahlreiche Arbeiten haben den Einfluss der thermischen Behandlung von Holz durch Sorptionsmessungen verfolgt (vgl. Kollmann und Schneider 1963). Da die Quellung von Lignocellulosen auch durch Bestimmung des Wasserrückhaltevermögens (WRV-Wert) erfasst werden kann, eine Methode, die seit mehreren Jahrzehnten für die Erfassung von Veränderungen in dem Quellvermögen von Zellstoffen herangezogen wird (vgl. Jayme und Büttel 1966), schien es von Interesse, festzustellen, inwieweit die Methode für die Erfassung der Veränderung in den Quellungseigenschaften von Holz durch thermische Behandlung verwendet werden kann.

\section{Material und Methodik}

Die Laubhölzer Esche (Fraxinus excelsior L.) und Buche (Fagus sylvatica L.) sowie das Nadelholz Fichte (Picea abies L.) wurden unter industriellen Versuchsbedingungen thermisch modifiziert. Nach der Modifizierung wurden aus den drei Hölzern Späne der Dimension (0,2 mm-0,8 mm) hergestellt. Zum Vergleich wurden ebenfalls Späne der entsprechenden Dimension aus den unbehandelten Hölzern in die Untersuchung einbezogen. Für die Späne wurde das Wasserrückhaltevermögen in Anlehnung an das Zellcheming Merkblatt (IV/3/57) nach Wasserlagerung für $72 \mathrm{~h}$ ermittelt. Die Bestimmung des Quellwertes nach dem Merkblatt (IV/3/57) beruht darauf, dass die mit einem Überschuss an Wasser versetzte Lignocellulose unter genormten Bedingungen gequollen und anschließend abzentrifugiert wird, hierdurch wird weitgehend lediglich Quellungswasser in der Zellwand zurückgehalten. 


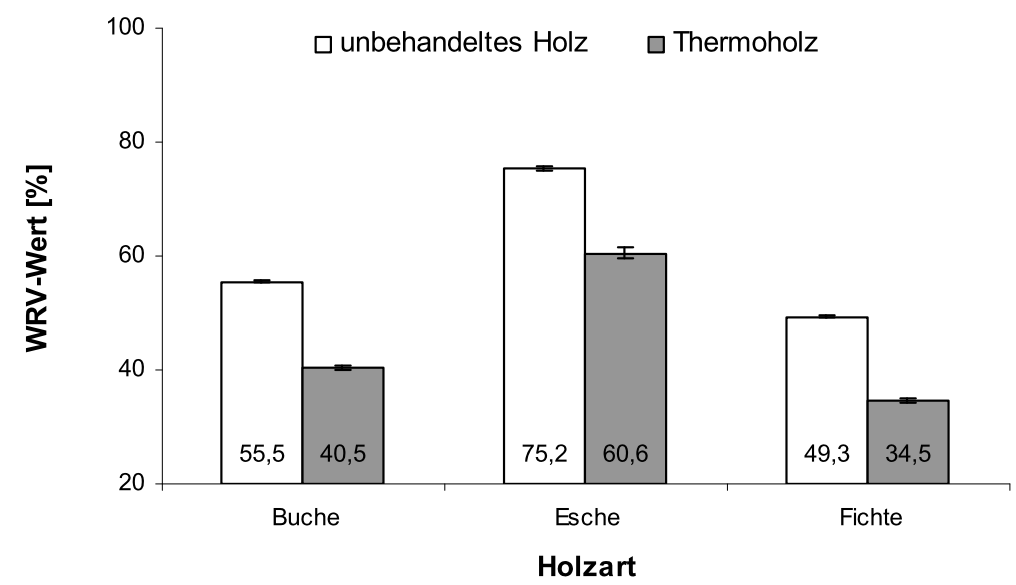

Abb. 1 Wasserrückhaltevermögen von Holzspänen $(0,2 \mathrm{~mm}-0,8 \mathrm{~mm})$ aus unbehandeltem Holz und Thermoholz der Buche, Esche und Fichte nach einer Wasserlagerung für $72 \mathrm{~h}$. Angegeben sind jeweils die Mittelwerte aus vier Einzelmessungen

Fig. 1 WRV-value of chips $(0.2 \mathrm{~mm}-0.8 \mathrm{~mm})$ prepared from untreated and thermally modified wood from ash, beech and spruce after storage in water for $72 \mathrm{~h}$. The results are the average values of four measurements

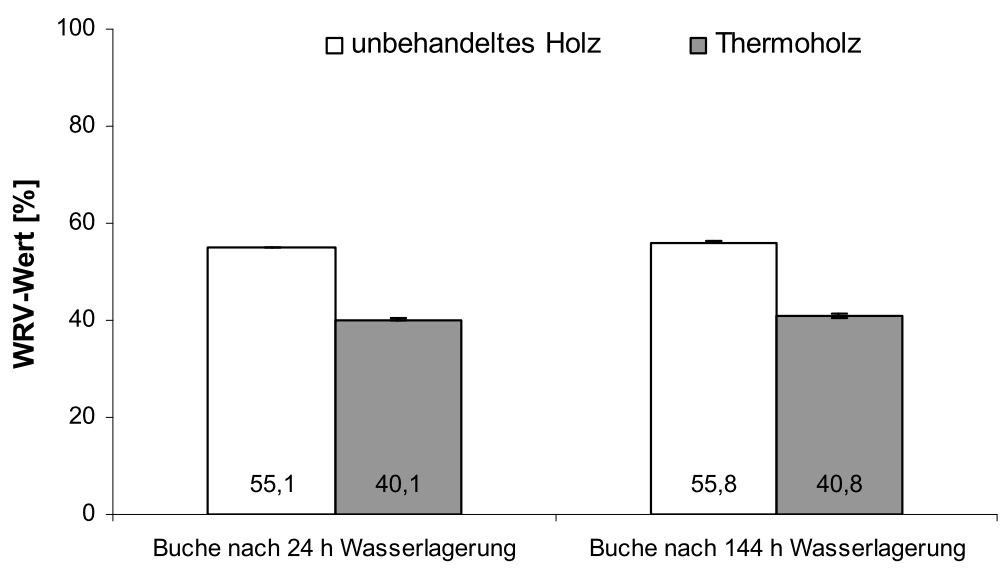

Abb. 2 Wasserrückhaltevermögen von Holzspänen $(0,2 \mathrm{~mm}-0,8 \mathrm{~mm})$ aus unbehandeltem Holz und Thermoholz der Buche nach einer Wasserlagerung für $24 \mathrm{~h}$ und $144 \mathrm{~h}$. Angegeben sind jeweils die Mittelwerte aus vier Einzelmessungen

Fig. 2 WRV-value of chips $(0.2 \mathrm{~mm}-0.8 \mathrm{~mm})$ prepared from untreated and thermally modified wood from beech after storage in water for $24 \mathrm{~h}$ and $144 \mathrm{~h}$. The results are the average values of four measurements

\section{Ergebnisse und Diskussion}

Die Ergebnisse der WRV-Bestimmungen sind in Abb. 1 für die drei Hölzer Buche, Esche und Fichte nach einer Lagerung in Wasser für $72 \mathrm{~h}$ zusammengestellt. Daraus wird deutlich, dass die thermische Modifizierung das Wasserrückhaltevermögen des Holzes herabsetzt. So nahm der WRV-Wert der Fichtenspäne von 49,3\% auf 34,5\% um ca. $30 \%$ ab und der von Buche von 55,5\% auf 40,5\% um ca. $27 \%$. Im Falle von Eschenholz war der Abfall des WRVWertes zwar deutlich (ca. 19\%) aber relativ gesehen weitaus geringer.

In weiteren Arbeiten wurde für Buchenholz die Lagerungsdauer zum einen auf $144 \mathrm{~h}$ verlängert und zum anderen auf $24 \mathrm{~h}$ verkürzt (Abb. 2). In beiden Fällen blieb der Unterschied im WRV-Wert zwischen dem thermisch behandelten und dem thermisch unbehandelten Holz annähernd unverändert.

Die Ergebnisse lassen den Schluss zu, dass es anscheinend zu einer ,irreversiblen Verhornung“ in der Zellwand infolge thermischer Behandlung kommt.

Open Access Dieser Artikel unterliegt den Bedingungen der Creative Commons Attribution Noncommercial License. Dadurch sind die nichtkommerzielle Nutzung, Verteilung und Reproduktion erlaubt, sofern der/die Originalautor/en und die Quelle angegeben sind.

\section{Literatur}

Ewert M, Scheiding W (2005) Thermoholz in der Anwendung - Eigenschaften und Möglichkeiten. Holztechnologie 46(2):22-29

Fengel D (1966a) Über die Veränderung des Holzes und seiner Komponenten im Temperaturbereich bis $200^{\circ} \mathrm{C}$. Teil $1-$ Heiß- und 
Kaltwasserextrakte von thermisch behandeltem Fichtenholz. Holz Roh- Werkst 24:9-14

Fengel D (1966b) Über die Veränderung des Holzes und seiner Komponenten im Temperaturbereich bis $200^{\circ} \mathrm{C}$. Teil 2 - Die Hemicellulosen in unbehandeltem und thermisch behandeltem Fichtenholz. Holz Roh- Werkst 24:98-109

Hill CAS (2006) Wood modification, chemical, thermal and other processes. Wiley, Chichester, S. 155-171
Jayme G, Büttel H (1966) Über die Bestimmung und Bedeutung des Wasserrückhaltevermögens (des WRV-Wertes) verschiedener gebleichter und ungebleichter Zellstoffe. Das Papier 20(7):357-365

Kollmann F, Schneider A (1963) Über das Sorptionsverhalten wärmebehandelter Hölzer. Holz Roh- Werkst 21:77-85

Windeisen E, Strobel C, Wegener G (2007) Chemical changes during the production of thermo-treated beech wood. Wood Sci Technol 41:523-536 